\title{
Educación inclusiva y digital: desafíos y propuestas a partir del COVID-19
}

Nuria Villa-Fernández ${ }^{1}$

Universidad Internacional de La Rioja nuria.villa@unir.net

Ángela Martín-Gutiérrez²

Universidad Internacional de La Rioja

Universidad de Sevilla

angela.martin@unir.net

DOI: https://doi.org/10.21158/2357514x.v8.n2.2020.2715

Cómo citar este artículo: Villa-Fernández, N.; Martín-Gutiérrez; Á. (2020). Educación inclusiva y digital: desafíos y propuestas a partir del COVID-19. Revista Virtu@Imente, 8(2), 7-27.

DOI: https://doi.org/10.21158/2357514x.v8.n2.2020.2715

Fecha de recepción: 08 de agosto de 2020

Fecha de aprobación: 06 de septiembre de 2020

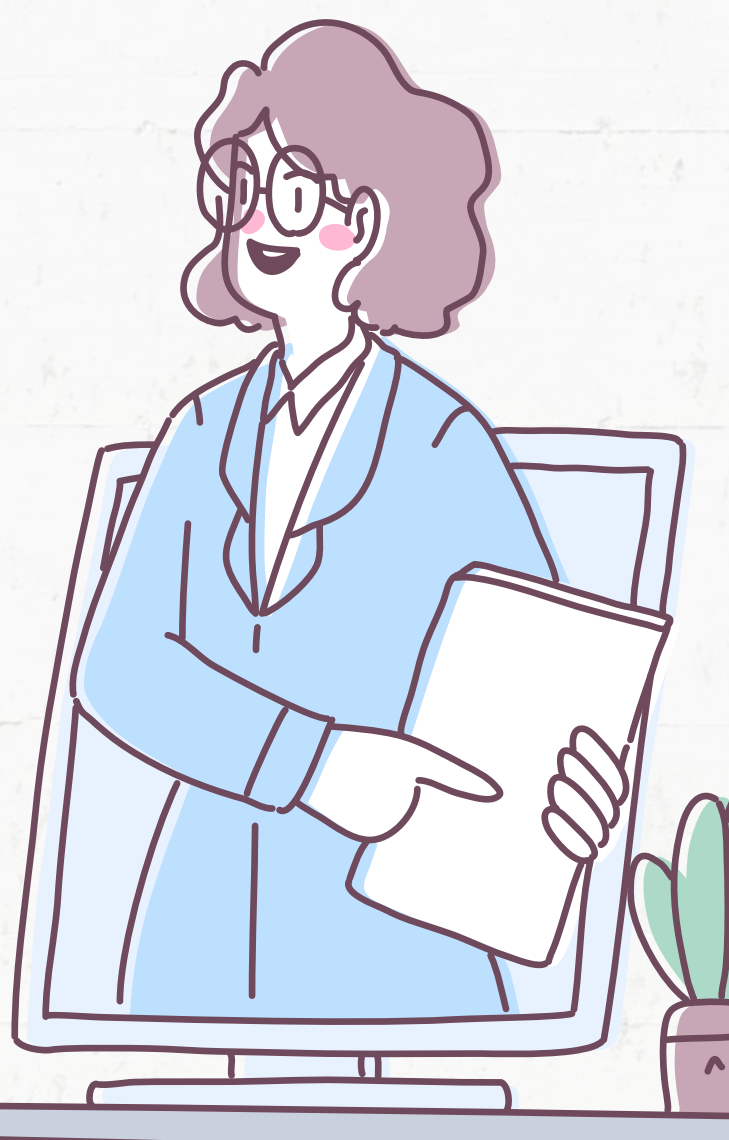

Licenciada en Pedagogía por la Universidad Complutense de Madrid (UCM). Doctora en Filosofía y Ciencias de la Educación por la Universidad Complutense de Madrid (UCM).

ORCID: https://orcid.org/0000-0002-8154-1129

Licenciada en Pedagogía por la Universidad de Sevilla (US). Máster Universitario en Dirección, Evaluación y Calidad de las Instituciones de Formación (US) y Doctora Internacional en Educación (US).

ORCID: https://orcid.org/0000-0001-9847-245X 


\section{RESUMEN}

Nos encontramos ante un desafío mundial sin precedentes en el ámbito social y, por tanto, educativo, con la crisis de la pandemia del COVID-19. En distintos estudios internacionales se reflejan las consecuencias que ha supuesto para el alumnado, profesorado y la familia el cierre de las instituciones educativas. En este sentido, se hace más que necesario el trabajo conjunto de toda la ciudanía para alcanzar una educación inclusiva y de calidad para todas las personas, sin excluir a nadie, lo que implica la cooperación de todos los sectores de la población. Por tal motivo, en este trabajo se presenta un análisis de las demandas socioeducativas actuales y se insiste en el valor de la pedagogía y de la formación de la comunidad educativa en el ámbito de la educación inclusiva y tecnológica como derecho. Se destacan algunas metodologías y estrategias favorecedoras de esta educación con mayúsculas, como el diseño universal para el aprendizaje, que suponen un reto para seguir trabajando por una sociedad más democrática y justa. El análisis demuestra que es necesario proyectar mejoras sociales y educativas, ya que la situación en la que nos encontramos perdurará un largo tiempo y seguirá haciendo visibles las nuevas realidades y las transformaciones necesarias que deben realizarse para que ningún colectivo quede excluido o discriminado, velando así por el cumplimiento efectivo de los derechos humanos.

Palabras clave: educación inclusiva; educación digital; educación tecnológica; Diseño Universal para el Aprendizaje (DUA); COVID-19; derechos humanos. 


\section{Inclusive and digital education: challenges and proposals from COVID-19}

\section{ABSTRACT}

With the COVID-19 pandemic crisis, we have been facing an unprecedented global challenge in the social sphere, and therefore, in the educational field. Various international studies reflect the consequences of closing educational institutions for students, teachers, and families. In this sense, it is more than necessary for all citizens to work together with the purpose of achieving inclusive and quality education for all people, excluding no one, which implies working with all the sectors of the population. For this reason, this work presents an analysis of the current socio educational demands, and insists on the value of pedagogy and the training of the educational community, as a right, in the field of inclusive education and technology. Some methodologies and strategies in favor of this education are highlighted with a capital letter, such as the universal design for learning, which represents a challenge to continue working for a more democratic and fair society. The analysis shows that it is necessary to project social and educational improvements, as the situation we are currently living will last for a long period and will continue to make visible the new realities and the transformations that must be made so that no group is excluded or discriminated, thus ensuring the effective fulfillment of human rights.

Keywords: inclusive education; digital education; technological education; Universal Design for Learning (UDL); COVID-19; human rights. 


\section{Educação inclusiva e digital: desafios e propostas do COVID-19}

\section{RESUMO}

Estamos diante de um desafio mundial sem precedentes no âmbito social e, portanto, educacional, com a crise da pandemia do COVID-19. Diferentes estudos internacionais refletem as consequências que supõem o fechamento das instituições de ensino para alunos, professores e famílias. Neste sentido, o trabalho em conjunto de todos os cidadãos é mais do que necessário para conseguir uma educação inclusiva e de qualidade para todas as pessoas, sem excluir ninguém, o que implica a cooperação de todos os setores da população. Por isso, este artigo apresenta uma análise das demandas socioeducativas atuais e insiste na valorização da pedagogia e na formação da comunidade educativa no campo da educação inclusiva e tecnológica como um direito. Algumas metodologias e estratégias que favorecem essa educação são destacadas com letras maiúsculas, como o Desenho Universal para a Aprendizagem, que representam um desafio para continuar trabalhando por uma sociedade mais democrática e justa. A análise mostra que é necessário projetar melhorias socioeducativas, já que a situação em que nos encontramos durará muito e continuará a tornar visíveis as novas realidades e as transformações necessárias que devem ser realizadas para que nenhum grupo seja excluído ou discriminado, garantindo assim um efetivo cumprimento dos direitos humanos.

Palavras-chave: educação inclusiva; educação digital; educação tecnológica; Desenho Universal para Aprendizagem (DUA); COVID-19; direitos humanos. 


\section{Éducation inclusive et numérique: défis et propositions tenant compte de la pandémie due au COVID-19}

\section{RÉSUMẼ}

Nous sommes aujourd'hui confrontés à un défi mondial sans précédent dans le domaine social et éducatif dû à la pandémie de COVID-19. Plusieurs études internationales analysent les conséquences, pour les étudiants, leurs familles et les enseignants de la fermeture des établissements d'enseignement. En ce sens, un travail conjoint de toutes les parties prenantes est plus que nécessaire pour parvenir à une éducation inclusive et de qualité, impliquant la coopération de tous les secteurs de la population. Cet article présente une analyse des nécessités socio-éducatives actuelles et insiste sur l'importance pédagogique et formative de la communauté éducative dans le domaine de l'éducation numérique et inclusive. Les méthodologies et stratégies favorisant ce type d'éducation seront mises en exergue pour permettre une conception universelle de l'apprentissage qui pose un défi pour l'emploi dans une société plus démocratique et plus juste. L'analyse montre qu'il est nécessaire de mettre en place des améliorations sociales et éducatives dans la mesure où la pandémie que nous connaissons risque de durer et d'impacter les nouvelles réalités et transformations nécessaires devant être apportées pour qu'aucun groupe social ne soit exclu ou discriminé et ainsi assurer le respect des droits humains.

Mots-cléfs: éducation inclusive; éducation numérique; éducation technologique; conception universelle pour l'apprentissage (CUA); COVID-19; droits humains. 


\section{Introducción}

La sociedad está sujeta a cambios acelerados y continuos, producidos por la globalización, los avances científicos y tecnológicos, la complejidad social, la difusión de las redes de comunicación, la multiculturalidad, la diversidad y los cambios laborales, entre otros. Actualmente, sumada a estos cambios, nos encontramos en una crisis internacional provocada por el virus del COVID-19, que afecta a todos los sectores — social, económico, educativo, etc.-. Así, el nuevo escenario al que nos enfrentamos implica un replanteamiento de los modos en los que se enseña y se aprende. Transformar el sistema educativo presencial en uno online supone un reto complejo para todas las personas, incluso para quienes ya estábamos familiarizados con él.

Desde este punto de vista, la perspectiva pedagógica resulta ser una pieza crucial, para diseñar métodos de enseñanza-aprendizaje (E-A) adaptados a las circunstancias, así como para ofrecer al profesorado recursos y estrategias más acordes con los entornos diversos en los que nos relacionamos, potenciando así una educación inclusiva y tecnológica para todas las personas.

\section{Objetivos}

Este trabajo aborda la educación inclusiva como derecho y la inclusión digital para afrontar los nuevos desafíos que nos plantea desde el ámbito socioeducativo el COVID-19. En esta línea, se ofrecen propuestas desde el aprendizaje a distancia, la accesibilidad y el Diseño Universal para el Aprendizaje (DUA) que favorezcan la educación para todo el alumnado y la eliminación de la exclusión social y digital.

\section{Demandas socioeducativas en tiempos complejos}

En el escenario actual que vivimos, la Organización de Naciones Unidas para la Educación, la Ciencia y la Cultura (Unesco) nos muestra el impacto de la pandemia del COVID-19 en la educación a en el mundo. La Unesco apoya a los países en sus esfuerzos por mitigar el impacto del cierre de escuelas, particularmente para las comunidades más vulnerables y desfavorecidas, y facilitar la continuidad de la educación para todos a través del aprendizaje a distancia.

En esta línea, el Informe de Seguimiento de la Educación en el Mundo 2020 (Global Education Monitoring Report Team, 2020), denominado «Inclusión y Educación: Todos sin Excepción», muestra que, en el mundo, las dimensiones de la discriminación por motivos de género, lejanía, riqueza, diversidad funcional -discapacidad-, etnia, idioma, migración, desplazamiento, encarcelamiento, orientación sexual, identidad y expresión de género, religión y otras creencias y actitudes, niega a bastantes estudiantes el derecho a ser educados y educadas con sus compañeros y compañeras, o a recibir una educación de la misma calidad. 
En el mapa interactivo de la página web de la Unesco (Figura 1) podemos hacer seguimiento a los cierres de escuelas en todo el mundo pinchando en cada uno de los países. Actualmente, 290 millones de estudiantes se encuentran sin clases y se advierte que esta situación puede aumentar las desigualdades sociales (Unesco, 2020a).

Figura 1. Mapa del seguimiento mundial de los cierres de escuelas causados por el COVID-19

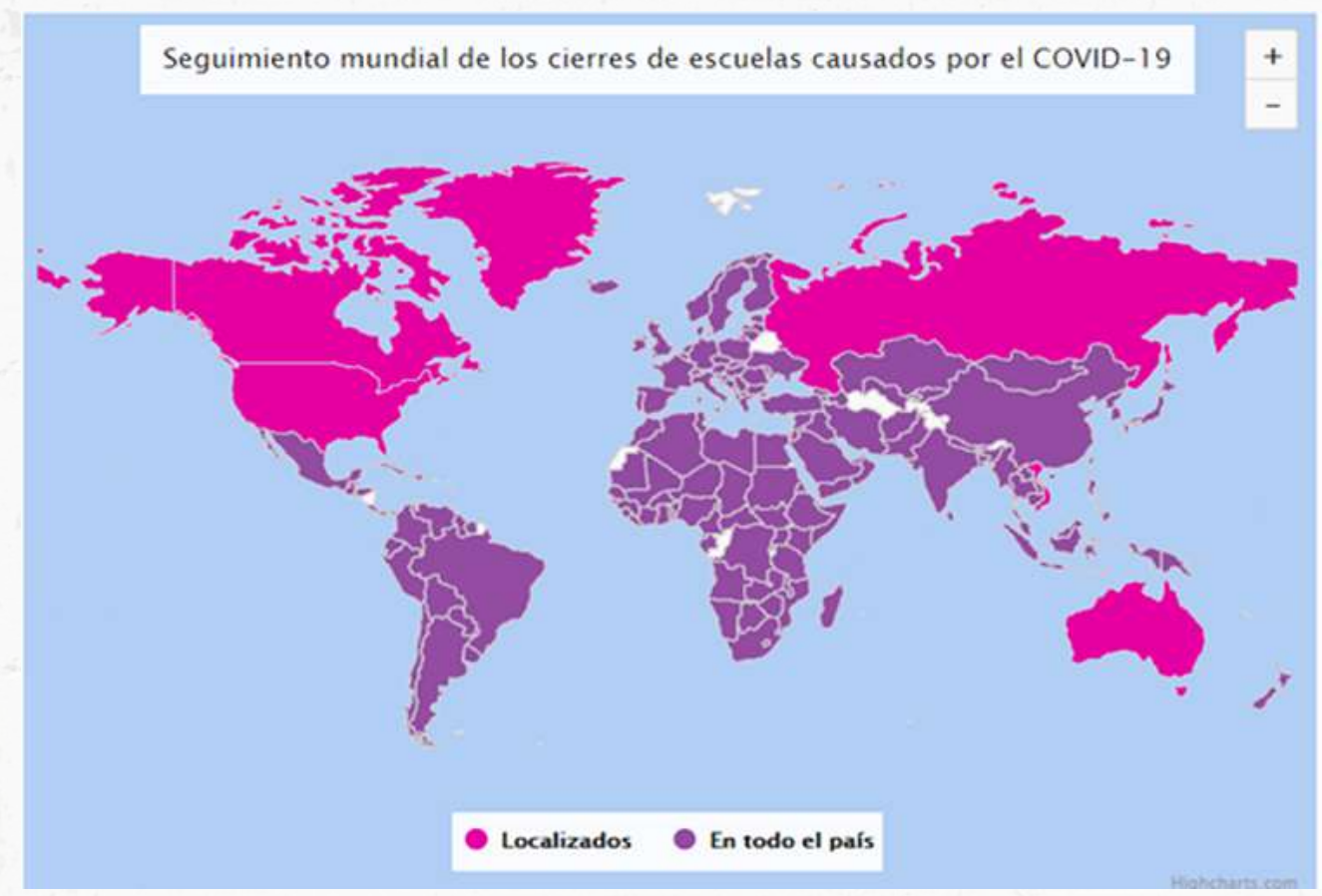

Fuente. Unesco, 2020a.

En el caso de España, los datos que arroja muestran que hay más de 9.706 .284 estudiantes afectados por el cierre de las escuelas debido al COVID-19. 
Figura 2. Estudiantes afectados por el cierre de escuelas en España por nivel educativo y género

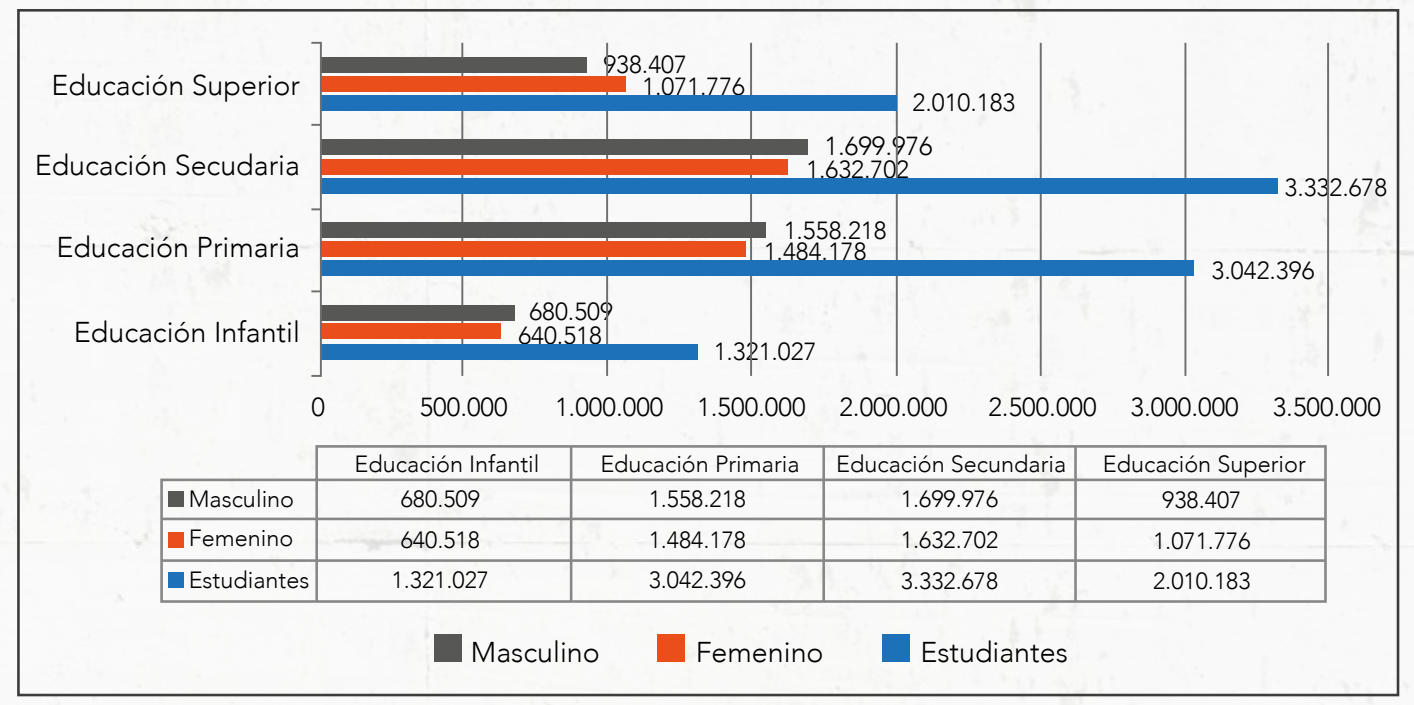

Fuente. Elaboración propia a partir de datos de la Unesco, 2020a.

Estos datos numéricos dan cuenta de los millones de niños, niñas y jóvenes que se han visto afectados por el cierre de sus escuelas, colegios, institutos y universidades en España; si añadimos a sus familias y maestros y maestras, la cifra de las personas a las que perturba esta situación se dispara.

Se hace necesario, por tanto, compatibilizar la vida familiar y escolar con la nueva realidad vivida, y la coordinación familia/ escuela se vuelve imprescindible. Para que esto sea posible, debemos de ver el confinamiento en nuestros hogares como un reto al que enfrentarnos y del que, sin duda, saldremos reforzados gracias a la implicación y actitud de todos y todas.
Al encontrarnos en un escenario educativo no presencial se requieren espacios virtuales que permitan la comunicación y el encuentro. Esto ha supuesto una gran transformación en la manera de relacionarnos, de enseñar y de aprender. Las personas más perjudicadas han sido quienes no tienen acceso a esa tecnología que facilita este encuentro, produciéndose una brecha digital. En este sentido, uno de los grandes revolucionarios en educación, que ha trabajado para la eliminación de la brecha digital con experimentos como «Un agujero en la pared» (1999), es Sugata Mitra, que demostró que los niños y niñas pueden aprender libremente, sin intervención adulta, gracias al internet y al trabajo cooperativo. 
El autor nos indica que, en ocasiones, «los mejores profesores y las mejores escuelas no están donde más se los necesita» (Mitra, 2010), por lo tanto, su deseo es construir una escuela en la nube, donde niños y niñas puedan explorar y aprender unos de otros. Así, creó los conceptos de «ambientes de aprendizaje autoorganizativos» (SOLE) y «ambientes mediados autoorganizativos» (SOME). De los experimentos concluyó que los y las estudiantes tenían mejores resultados al tener a un adulto que motivara y aconsejara, planteándoles una pregunta para que investigaran en grupo. Se observaron resultados que podrían revolucionar lo que pensamos acerca de la enseñanza, sustituyendo «la amenaza» del examen por el placer de aprender (Mitra, 2016).

Ya Ferrández (2000, p. 3) expresaba que la educación era «un todo mágico y siempre inacabado». Por lo tanto, la esperanza tiene que estar presente y, como diría Freire (2001): «Al repensar los datos concretos de la realidad vivida, el pensamiento profético, que es también utópico, implica la denuncia de cómo estamos viviendo y el anuncio de cómo podríamos vivir. Por eso mismo es un pensamiento esperanzador» (p. 131). Y continúa diciéndonos que la naturaleza humana se construye social e históricamente.

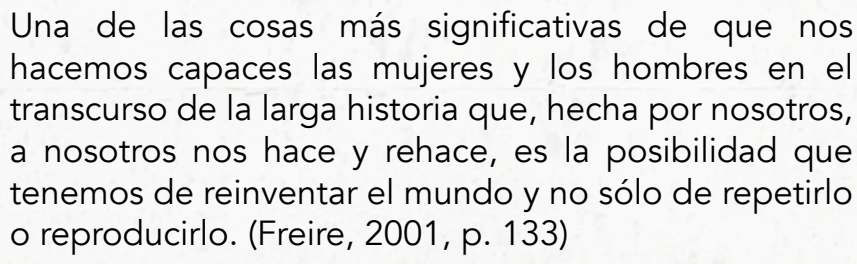

Para que esto sea posible, hemos de cambiar nuestra forma de pensar y empezar a considerar cómo podemos contribuir a la construcción del capital social (Calvo, 2008), ya que ofrece beneficios a las personas y a las comunidades a las que pertenecen. Por ello, es necesario crear redes en el ámbito social, para promoverlo y mejorarlo (Díaz y Civís, 2011), con la finalidad de prevenir situaciones de conflicto, entablar procesos de socialización u otros que persigan la cohesión y participación en el desarrollo de procesos, y la vinculación con las metas y proyectos compartidos (Azqueta, Gavaldón y Margalef, 2007).

Ligadas al concepto del "capital social», se encuentran las teorías de la estructuración y del aprendizaje situado. La teoría de la estructuración no se enfoca en el actor individual o la sociedad en su totalidad, sino en las prácticas sociales desarrolladas a través del tiempo y el espacio (Lois, 2011). Autores como Levine y Marcus (2010) muestran cómo la construcción del conocimiento práctico se sustenta en los procesos de interacción social. Por ejemplo, adoptando estrategias interculturales, el desarrollo de procesos de inclusión de la sociedad civil en los procesos de deliberación, la práctica de modelos de evaluación cualitativa del aprendizaje del alumnado y la formación del profesorado basada en el aprendizaje permanente mediatizado por la reflexión sobre la práctica y en ella mediante redes de democracia deliberativa $u$ otras estrategias compatibles con el desarrollo de interacciones basadas en procesos de colaboración y cooperación en la sociedad de la información y el conocimiento (SIC).

El aprendizaje situado también cobra especial importancia en este contexto, pues es el resultado de la actividad, del entorno y de la cultura social en la que se produce (GarcíaCarrasco, Gallardo, García-Manzano y Sánchez, 2012). Por lo que las actividades que se diseñan y desarrollan, y más en estos 
momentos, han de tener en cuenta esta realidad, originando un cambio en las creencias, actitudes y comportamientos por parte de las personas (Casquero, 2013). Como indican Martín, Rinaudo y Ordoñez (2012), cuando nos sentimos parte de nuestra comunidad nos volvemos más activos, involucrados y comprometidos con la cultura social, lo que hace que el significado del aprendizaje, progresivamente, se vaya configurado a través de lo que Lave y Wenger (1991) denominaban "participación periférica legítima»; es decir, aquella relación generada entre las personas recién llegadas y las más antiguas en una comunidad, con respecto a sus actividades, identidades, recursos y grupos de conocimiento y práctica.

En definitiva, el aprendizaje y, en consecuencia, las personas progresamos a través de la colaboración y de la construcción social del conocimiento. En este sentido, el compromiso social surge como el pilar en el que se sustenta el aprendizaje, ya que constituye la base de las políticas educativas, para contribuir a la mejora y al progreso social, sin olvidarse de aunar la equidad y la calidad (Santos, 2009).

Por tanto, la SIC y las Tecnologías de la Información y Comunicación (TIC) traen consigo nuevas exigencias para el ámbito educativo, pero también nuevas oportunidades para enseñar y aprender en contextos cambiantes (MartínGutiérrez y Morales-Lozano, 2013); así como nuevos modelos pedagógicos virtuales, que sean más inclusivos para todos y todas, en especial, para los grupos más discriminados, como son las personas con diversidad funcional (Villa, 2015).
En este sentido, la Unesco (2020b) lanza una "Coalición mundial para mejorar el aprendizaje a distancia», a raíz del cierre de las instituciones educativas para detener la propagación del COVID-19. Nuestros niños y niñas están aprendiendo de forma remota desde casa y esta situación supone un gran desafío para todos los implicados en el proceso educativo alumnado, profesionales de la educación y familias-.

Estos nuevos escenarios, a los cuales no estamos habituados, requieren de un fuerte componente motivacional intrínseco y de una colaboración conjunta para superar las barreras de la enseñanza y el aprendizaje ocasionadas por esta pandemia. Sin embargo, estos factores no son los únicos implicados; resulta necesaria la formación pedagógica en competencias digitales para superar los retos que nos plantea esta nueva situación en los ámbitos educativo, laboral, social, cultural y tecnológico, entre otros, que responda a las necesidades de toda la población en su conjunto. Esto sumado a otra de las barreras encontradas que es la accesibilidad a los recursos tecnológicos; por este motivo, en España, desde los ministerios, Consejerías de Educación, colegios, universidades, asociaciones, etc., se han impulsado iniciativas para dotar de medios informáticos al alumnado, así como de conectividad a sus hogares.

Ya se han producido acciones por parte del sector educativo en las que los profesionales de la educación han dirigido mensajes de aliento a sus estudiantes y familias a través de vídeos, canciones y cuentos, entre otros. Los niños y niñas no se han quedado atrás, y han respondido de la misma manera, con retos, aplausos colectivos y mensajes virtuales de felicitación. 


\section{Educación inclusiva como derecho}

Llegados a este punto, resulta imprescindible partir del derecho a la educación, recogido en la Declaración Universal de los Derechos Humanos y en la Convención de Derechos de las Personas con Discapacidad (ONU, 2006), para entender la educación inclusiva. Una educación de calidad tiene que ir de la mano con una educación inclusiva; para ello, el personal docente debe conocer y llevar a cabo estrategias y metodologías favorecedoras para todas las personas. En palabras de Echeita y Ainscow (2011), la inclusión busca la presencia, la participación y el éxito de todo el alumnado, precisa la identificación y la eliminación de barreras, y es un proceso de mejora constante.

La diversidad, en sentido amplio, es lo que nos hace positivamente diferentes. La forma en que entendamos dicha diversidad es muy importante. Desde la educación inclusiva e intercultural, la diferencia se presenta como un valor (Tonucci, 2017) con posibilidades de enriquecernos mutuamente como personas. El foco de esta mirada está en respetar la diversidad y el derecho a una educación inclusiva, y en crear ambientes de aprendizaje donde todas las personas puedan aprender, sean cuales sean sus características y necesidades. Desde la educación inclusiva se pretende eliminar las barreras que, debido a las múltiples fuentes de diversidad, ponen a una parte del alumnado en una situación de vulnerabilidad, provocándole diferentes modalidades de exclusión (Escudero, 2005).
Por tanto, como nos señala Vega (2010), la educación inclusiva es un deber de justicia y no es posible sin un profundo compromiso social. En este sentido, Slee (2012) habla de la escuela extraordinaria como aquella que trata de no diferenciar entre la escuela ordinaria y la especial, procurando no segregar ni crear «guetos» entre su alumnado, y considera la diversidad un proceso de enriquecimiento para toda la comunidad. "La educación inclusiva no es, en consecuencia, una formulación de ideas acerca de las necesidades educativas especiales, sino una teoría y una táctica de la reforma educativa y social» (Slee, 2012). Con el mismo propósito, surge en España la Plataforma Estatal por una Escuela Extraordinaria (2019), cuyo manifiesto defiende el derecho a la educación inclusiva como paso imprescindible hacia una sociedad inclusiva, cohesionada, que respete la diversidad y garantice los derechos de todas las personas.

Según la Unesco (2008), a través de la educación inclusiva, entendida como proceso de cambio y mejora, se favorecerá la atención a todo el alumnado, independientemente de sus necesidades, al apostar por una mayor participación en el aprendizaje y favorecerla tanto en el ámbito educativo como en el social. En este sentido, cabe destacar la importancia que tiene también la inclusión digital (digital inclusion), entendida como la participación plena de la ciudadanía, en igualdad de condiciones, en la sociedad del conocimiento, garantizando tanto el acceso a las nuevas tecnologías, a través de programas 
de ayudas a la infraestructura, como mediante la asunción, aplicación y promoción de los estándares y directrices de accesibilidad; y, naturalmente, mediante la formación y la educación (Gutiérrez, 2001).

Desde el «Index for Inclusion» realizado por Booth y Ainscow (2002) se ofrece un conjunto de materiales para apoyar a las escuelas en el proceso de desarrollo de una escuela inclusiva. Es un recurso muy enriquecedor para la formación del personal docente y es por ello por lo que desde aquí se anima a su utilización. Dicha guía cuestiona la atención a la diversidad de los centros en relación con las barreras que provocan necesidades de aprendizaje y de participación de todo su alumnado. Las tres dimensiones del índex son: establecer culturas inclusivas, políticas inclusivas y desarrollar prácticas inclusivas. Cada dimensión se divide en dos secciones: "Dimensión A»: crear culturas inclusivas, construir comunidad y establecer valores inclusivos; «Dimensión B»: establecer políticas inclusivas, desarrollar un centro escolar para todos y organizar el apoyo a la diversidad; "Dimensión C»: desarrollar prácticas inclusivas, construir un currículum para todos y orquestar el aprendizaje (Booth y Ainscow, 2015, p. 17).

Compartimos la reflexión que aporta Carbonell, cuando nos indica que:

Todos los teóricos de la inclusión argumentan que la educación inclusiva no es educación especial y no debe tomarse como referencia. Supone una superación del concepto de integración, muestra el mejor camino para superar cualquier tipo de discriminación y exclusión, implica un reconocimiento del derecho a una educación democrática y de calidad, tiene que ver con la reforma educativa y con la transformación escolar; por lo tanto, exige cambios fundamentales en el pensamiento educativo sobre el alumnado, en el currículo, la pedagogía y la organización escolar". (2015, pp. 127-128)

De esta manera, la inclusión trata de establecer las bases para que la escuela pueda educar con éxito y colaborar en la erradicación de las desigualdades e injusticias sociales. En esta línea, la educación inclusiva constituye hoy en día uno de los objetivos fundamentales de la agenda política global establecida para el año 2030 en Incheon (Unesco, 2016), lo que sin duda plantea un importante reto para los sistemas educativos. Para ello, es necesario cambiar diferentes aspectos del sistema educativo y, por ende, en la formación de toda la comunidad educativa, como una visión crítica de mejora de los contenidos, los enfoques, las estructuras, las estrategias y la evaluación, entendiendo que todo el alumnado forma parte del proceso y debe tener la posibilidad de construir un aprendizaje de calidad, sobre todo, aquellos que, por diferentes razones, están excluidos o en riesgo de ser marginados.

En esta línea, la Unesco (2020b) colabora con los ministerios de Educación de los países afectados por el COVID-19 e interesados, con el objetivo de garantizar la continuidad del aprendizaje para todos los niños y jóvenes, y hacer un seguimiento de este. Para ello, entre sus respuestas globales, se encuentra la citada Coalición Mundial para la Educación, con el propósito de ayudar a los países a ampliar las mejores soluciones de aprendizaje a distancia y llegar a los niños y jóvenes que corren mayor riesgo. Así, el 10 de marzo de 
2020 se programaron reuniones virtuales entre ministros para el aprendizaje de políticas sobre los esfuerzos para iniciar y ampliar las respuestas al cierre de las escuelas.

\section{Esta coalición también proporciona:}

- Asistencia técnica para preparar y desplegar con rapidez soluciones inclusivas de aprendizaje a distancia, utilizando técnicas de alta y baja tecnología, así como métodos tradicionales.

- Seminarios web para funcionarios del Ministerio de Educación y otras partes interesadas en compartir información sobre los esfuerzos de los países para mantener la provisión de educación inclusiva en diferentes contextos.

- Selección de recursos pedagógicos digitales que los gobiernos, centros escolares, docentes y familias pueden utilizar para proponer soluciones a los alumnos y alumnas.

- Repertorio de plataformas nacionales de aprendizaje para favorecer la continuidad del programa escolar.

- Alianzas para ampliar las capacidades nacionales y locales que aseguren la provisión remota de educación.

La Unesco facilita la siguiente dirección de correo electrónico, para que los y las representantes de los países que deseen unirse se comuniquen por esta vía: COVID19taskforce@unesco.org.

\section{El Diseño Universal para el Aprendizaje como estrategia metodológica para la inclusión y el respeto a la diversidad}

Dentro de la situación actual que estamos viviendo en el ámbito educativo con la crisis del COVID-19, los maestros y las maestras siguen teniendo un papel fundamental para lograr la mediación entre las necesidades del alumnado, las familias y la sociedad, al intentar construir con sus alumnos y alumnas valores y competencias sociales basados en la inclusión y el respeto a la diversidad.

En este punto se hace necesario referir que la inclusión del alumnado dependerá fundamentalmente de que todos y todas, pero, en especial el profesorado, tome conciencia de la influencia que ejercemos en el alumnado, como seres humanos y como profesionales, con valores y actitudes, y con un serio y riguroso compromiso en la educación desarrollada en contextos de diversidad (Leiva, 2012). Si este planteamiento es fundamental para la educación presencial, más aún lo es, si cabe, en contextos no presenciales o virtuales, que requieren humanizarse para que las distancias originadas se acorten, mediante la motivación y recursos adecuados que favorezcan el aprendizaje de todo el alumnado. 
Para que exista una adecuada concienciación y sensibilización por parte del profesorado por la inclusión y, por tanto, por los derechos a la educación de los niños y las niñas, es necesario dotarlo de una formación pedagógica y tecnológica adecuada, como un elemento de construcción de los aprendizajes y de crecimiento personal. Es decir, en la formación del profesorado debe primar la reflexión en y sobre la acción, apostando por un modelo docente que se caracterice por su compromiso con la educación de todo el alumnado, así como por su interés por investigar e indagar sobre nuevas prácticas y estrategias, en situaciones cambiantes como la que estamos viviendo.

No obstante, el profesorado no debe intervenir individualmente; como indica Arroyo (2013), es importante que trabaje de manera colaborativa, favoreciendo así el intercambio de experiencias y estableciendo, al mismo tiempo, un ejercicio de planificación y ayuda mutua que contribuya a mejorar la conciencia de la comunidad educativa. Como hemos destacado en apartados anteriores, la educación inclusiva es un reto ante la diversidad del alumnado y nuestra preocupación se debe de centrar en cómo podemos enseñar juntos a alumnos y alumnas heterogéneos, con motivaciones, intereses y características diferentes.
En este sentido, el Diseño Universal para el aprendizaje (DUA) resulta ser la mejor estrategia en los nuevos escenarios educativos, ya que se torna como favorecedora de una educación para todas las personas. Es un marco basado en la investigación para el diseño de un currículum —esto es, objetivos educativos, métodos, materiales y evaluacionesque les permita a todas las personas adquirir conocimiento, habilidades y motivación para aprender. Se apoya en tres principios básicos: utilizar diferentes formas de expresión, de implicación y de presentar la información, en su aplicación a todo el currículo. El DUA asume que todas las personas presentan diferencias en tres aspectos sociocognitivos: en el reconocimiento de lo que nos rodea, en las respuestas que damos física y cognitivamente, y en la apreciación afectiva y la valoración de lo que nos rodea.

Bajo este modelo, y como se indica en la figura 3, se proponen tres principios fundamentales (CAST, 2018): proporcionar múltiples formas de compromiso —el porqué del aprendizaje-, proporcionar múltiples medios de representación —el qué del aprendizaje-y proveer múltiples formas de acción y expresión -el cómo del aprendizaje-. 
Figura 3. Diseño Universal para el Aprendizaje: principios, pautas y puntos de verificación del marco del DUA

\begin{tabular}{|c|c|c|c|}
\hline & $\begin{array}{l}\text { Proporcionar múltiles formas de } \\
\text { implicación }\end{array}$ & $\begin{array}{l}\text { Proporcionar múltiles formas de } \\
\text { representación }\end{array}$ & $\begin{array}{l}\text { Proporcionar múltiles formas de } \\
\text { acción y expresión }\end{array}$ \\
\hline Pautas & $\begin{array}{c}\text { Proporcionar opciones para captar el } \\
\text { interés (7) }\end{array}$ & $\begin{array}{l}\text { Proporcionar opciones para } \\
\text { la recepción (1) }\end{array}$ & $\begin{array}{l}\text { Proporcionar opciones para la } \\
\text { interacción física (4) }\end{array}$ \\
\hline \multirow{3}{*}{$\begin{array}{l}\text { Puntos de } \\
\text { verificación }\end{array}$} & $\begin{array}{l}\text { Optimizar la elección inividual y la } \\
\text { autonomía (7.1) }\end{array}$ & $\begin{array}{c}\text { Ofrecer opciones para la modificación y } \\
\text { personalización en la presentación de la } \\
\text { información (1.1) }\end{array}$ & $\begin{array}{c}\text { Variar los métodos para la respuesta y la } \\
\text { navegación (4.1) }\end{array}$ \\
\hline & $\begin{array}{c}\text { Optimizar la relevancia, el valor y la } \\
\text { autenticidad (7.2) }\end{array}$ & $\begin{array}{c}\text { Ofrecer alternativas para la información } \\
\text { auditiva (1.2) }\end{array}$ & $\begin{array}{c}\text { Optmizar el acceso a las herramientas y los } \\
\text { productos y tecnologías de apoyo (4.2) }\end{array}$ \\
\hline & $\begin{array}{c}\text { Minimizar la sensación de inseguridad y } \\
\text { distracciones (7.3) }\end{array}$ & $\begin{array}{c}\text { Ofrecer alternativas para la información visual } \\
(1.3)\end{array}$ & \\
\hline Pautas & $\begin{array}{c}\text { Proporcionar opciones para mantener el } \\
\text { esfuerzo y la persistencia (8) }\end{array}$ & $\begin{array}{c}\text { Proporcionar opciones para el lenguage, } \\
\text { las expresiones matemáticas y los } \\
\text { símbolos (2) }\end{array}$ & $\begin{array}{l}\text { Proporcionar opciones para la expresión } \\
\text { y comunicación (5) }\end{array}$ \\
\hline \multirow{5}{*}{$\begin{array}{l}\text { Puntos de } \\
\text { verificación }\end{array}$} & $\begin{array}{c}\text { Resaltar la relevancia de las metas y los } \\
\text { objetivos (8.1) }\end{array}$ & Clarificar el vocabulario y símbolos (2.1) & $\begin{array}{c}\text { Utilizar múltiples medios de comunicación } \\
(5.1)\end{array}$ \\
\hline & $\begin{array}{c}\text { Variar los niveles de exigencia y recursos para } \\
\text { optimizar los desafios (8.2) }\end{array}$ & Clarificar la sintáxis y la escritura (2.2) & $\begin{array}{l}\text { Usar múltiples herramientas para la } \\
\text { construcción y la composición (5.2) }\end{array}$ \\
\hline & $\begin{array}{l}\text { Fomentar la colaboración y la comunidad } \\
\qquad(8.3)\end{array}$ & $\begin{array}{c}\text { Facilitar la decodificación de textos, } \\
\text { anotaciones matemáticas y símbolos (2.3) }\end{array}$ & $\begin{array}{l}\text { Definir competencias con niveles de apoyo } \\
\text { graduados para la práctica y ejecución (5.3) }\end{array}$ \\
\hline & $\begin{array}{c}\text { Utilizar el feedback orientado hacia la } \\
\text { maestría en una tarea (8.4) }\end{array}$ & $\begin{array}{c}\text { Promover la comprensión entre diferentes } \\
\text { idiomas (2.4) }\end{array}$ & \\
\hline & & $\begin{array}{c}\text { Ilustrar las ideas principales a través de } \\
\text { múltiples medios (2.5) }\end{array}$ & \\
\hline Pautas & $\begin{array}{l}\text { Proporcionar opciones para la } \\
\text { autorregulación (9) }\end{array}$ & $\begin{array}{l}\text { Proporcionar opciones para la } \\
\text { comprensión (3) }\end{array}$ & $\begin{array}{l}\text { Proporcionar opciones para las funciones } \\
\text { ejecutivas ( } 3 \text { ) }\end{array}$ \\
\hline
\end{tabular}




\begin{tabular}{|c|c|c|c|}
\hline \multirow{4}{*}{$\begin{array}{l}\text { Puntos de } \\
\text { verificación }\end{array}$} & $\begin{array}{c}\text { Promover expectativas y creencias que } \\
\text { optimizan la motivación (9.1) }\end{array}$ & Activar los conocimientos previos (3.1) & Guiar el establecimietno de metas (6.1) \\
\hline & $\begin{array}{c}\text { Facilitar estrategas y habilidades personales } \\
\text { para afrontar los problemas de la vida } \\
\text { cotidiana (9.2) }\end{array}$ & $\begin{array}{c}\text { Destacar patrones, características } \\
\text { fundamentales, ideas principales y relaciones } \\
\text { entre ellos (3.2) }\end{array}$ & $\begin{array}{c}\text { Apoyar la planificación y el desarrollo de } \\
\text { estrategias (6.2) }\end{array}$ \\
\hline & $\begin{array}{c}\text { Desarrollar la autoevaluación y la reflexión } \\
\qquad(9.3)\end{array}$ & $\begin{array}{c}\text { Guiar el procesamiento de la información, la } \\
\text { visualización y la manipulación (3.3) }\end{array}$ & $\begin{array}{l}\text { Facilitar la gestión de información y de } \\
\text { recursos (6.3) }\end{array}$ \\
\hline & & $\begin{array}{c}\text { Maximizar la memoria, transferencia y } \\
\text { generalización (3.4) }\end{array}$ & $\begin{array}{c}\text { Aumentar la capacidad para hacer un } \\
\text { seguimiento de los avances (6.4) }\end{array}$ \\
\hline Objetivos & Estudiante motivado y decidido & $\begin{array}{c}\text { Aprendiz capaz de identificar los recursos } \\
\text { adecuados }\end{array}$ & Estudiante orientado a cumplir metas \\
\hline
\end{tabular}

Fuente. CAST, 2018. Traducción de Educadua.

Por tanto, el DUA plantea un cambio actitudinal. Hay que actuar sobre el contexto para que los métodos instruccionales, los recursos, los materiales y la evaluación sean accesibles para todas las personas, partiendo desde el diseño, bajo una mirada inclusiva. Por eso, es necesario que dicho perfil profesional sea especialista en el DUA (Márquez, 2018; Pastor, 2018).

Es de gran interés en este sentido la Rueda DUA 2020 (Márquez, 2018), que aporta diversas aplicaciones, páginas web y recursos, que han sido elegidos para propiciar la participación de todo el alumnado en el aula (Figura 4). Como nos señala Antonio Márquez:
Posiblemente existan multitud de aplicaciones y recursos que hubiesen podido aportarse para enriquecer esta Rueda del DUA, sin embargo, se han seleccionado algunos que representan las posibilidades, casi infinitas, que nos ofrecen las tecnologías para apoyar el proceso de diseño universal y la eliminación de barreras en los procesos de E-A. Debe entenderse esta rueda como facilitadora de la inclusión y de uso general para diseñar actividades del aula, ofreciendo múltiples formas de participación. 
Figura 4. Rueda del DUA 2020

\section{RUEDA DEL DISEÑO UNIVERSAL PARA EL APRENDIZAJE 2020}

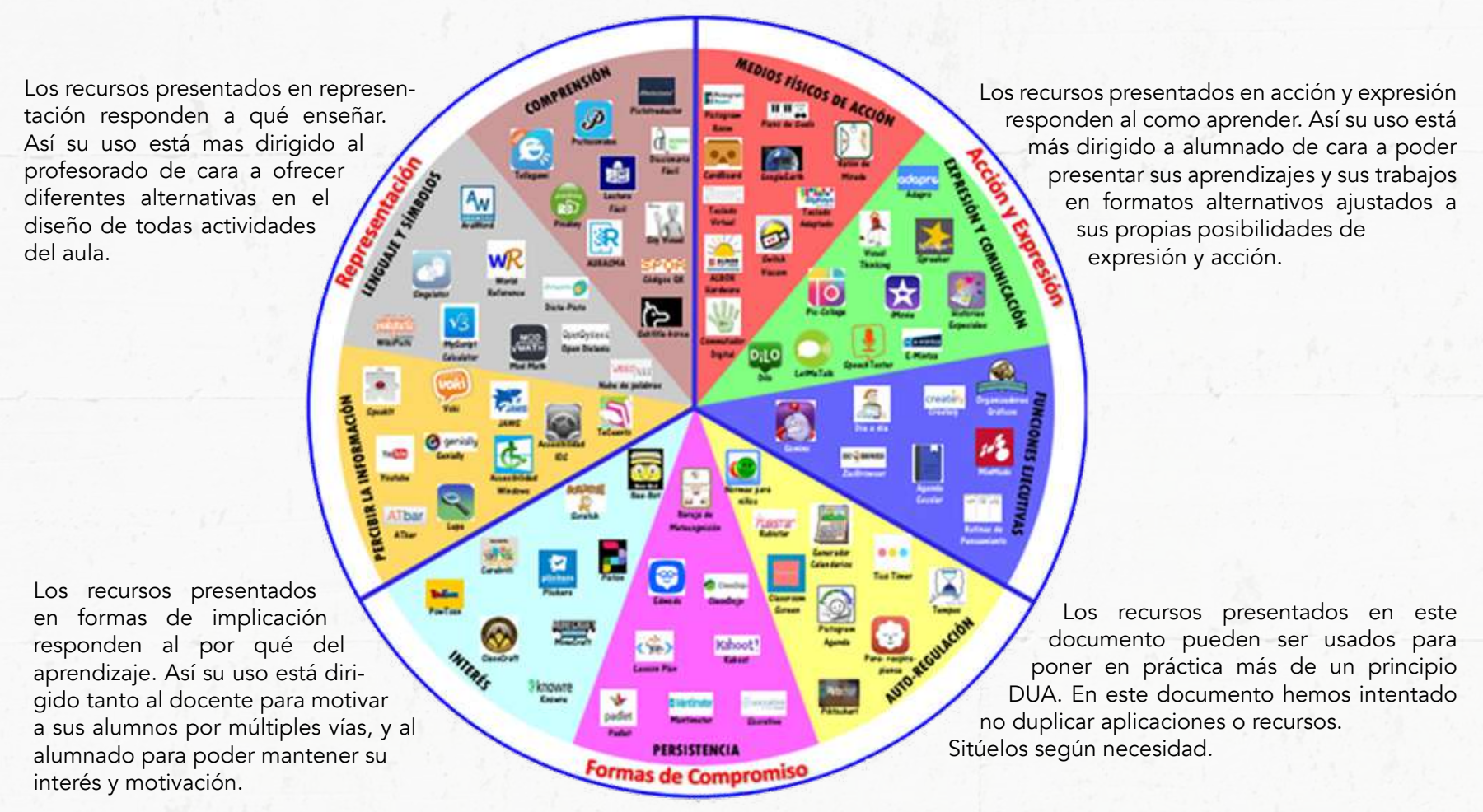

Fuente. Márquez, 2018. 
Por último, nos parece relevante destacar el proyecto «Formación curricular en diseño para todas las personas», desarrollado por el Real Patronato sobre Discapacidad y la Fundación de la Organización Nacional de Ciegos Españoles (ONCE), con la colaboración de la Conferencia de Rectores de las Universidades Españolas (CRUE) y la Agencia Nacional de Evaluación de la Calidad y Acreditación (ANECA), que busca apoyar la labor universitaria a la hora de introducir los conceptos de «accesibilidad universal» y «diseño para todas las personas» en los currículos formativos de las universidades. El planteamiento general es que todas las titulaciones que guardan alguna relación con el ser humano, el medio ambiente y el diseño del entorno físico y virtual han de incluir, en mayor o menor medida, conocimientos sobre estas cuestiones en sus planes de estudio.

Los materiales elaborados en las diferentes titulaciones, recogidos en la página web de la CRUE (2017), han sido los siguientes: 2010: Arquitectura, Diseño, Informática, Telecomunicaciones, Ingeniería de Caminos, Canales y Puertos, e Ingeniería Industrial; 2014: Administración y Dirección de Empresas, Derecho, Medicina, Pedagogía, Psicología y Trabajo Social; 2016: Ciencias Políticas, Enfermería, Farmacia, Periodismo, Sociología y Terapia Ocupacional; 2017: Educación —se incluyen los estudios en Educación Infantil, Educación Primaria, Educación Social y Máster del Profesorado-y Turismo. El objetivo final es formar a profesionales capaces de construir una sociedad inclusiva que garantice la igualdad de oportunidades. Busca el cumplimiento de la Ley Orgánica 4/2007, del 12 de abril, que modifica la Ley Orgánica de Universidades (Lomlou), que en su Disposición Adicional Vigésimo Cuarta, apartado 5, señala:
Todos los Planes de Estudios propuestos por las universidades deben tener en cuenta que la formación en cualquier actividad profesional debe realizarse desde el respeto y la promoción de los Derechos Humanos y los principios de Accesibilidad Universal y Diseño para Todos.

\section{Conclusiones}

En los escenarios vitales como en el que nos encontramos, se plantean demandas y desafíos sociales y educativos motivados por los cambios acelerados propios del mundo globalizado en el que convivimos. Y si a esta realidad le añadimos la crisis mundial sanitaria provocada por el COVID-19, sin duda alguna se hace más que necesaria la respuesta conjunta de toda la sociedad para lograr un mundo más humano y justo, $y$, por lo tanto, sin desigualdades.

La educación juega un papel clave en todo este proceso, ya que es uno de los derechos humanos básicos para alcanzar una mayor igualdad de oportunidades reales entre todas las personas, en tanto contribuye a nuestro desarrollo personal y social. Partiendo de esta premisa, la educación debe de responder a este reto, aun suponiendo un esfuerzo colectivo, ya que nos insta a cambiar los modelos de E-A tradicionales. Esto implica trasladarnos a nuevos escenarios de educación a distancia, que requieren de una formación pedagógica y tecnológica, y un compromiso por parte de todas las personas involucradas en el proceso educativo, que a veces resulta complicado llevar a la práctica. 
No obstante, este trabajo busca ofrecer una visión esperanzadora, aportando estrategias que se están llevando a cabo a nivel macro, como las facilitadas por la Unesco, y a nivel micro, como el DUA, para que apostemos por una verdadera educación inclusiva, eliminando barreras, afrontando estos cambios con motivación y aprendiendo de todo lo vivido.

Como señala Audrey Azoulay, directora general de la Unesco (2020b):

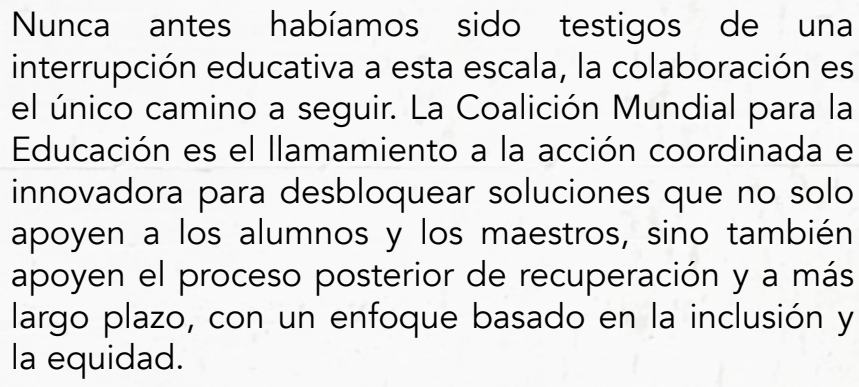

Como hemos visto, la situación actual debe servirnos como un punto de partida dentro de un proceso de reflexión educativa, donde todos los agentes involucrados en la educación repensemos los enfoques tradicionales en los que trabajamos, que parecen favorecer a un alumnado homogéneo, sin tener en cuenta la riqueza que la diversidad nos aporta y vulnerando, por tanto, los derechos de los grupos minoritarios y más desfavorecidos. De esta manera, debemos aunar nuestros esfuerzos a partir de ahora para diseñar y planificar nuevos enfoques y sistemas de educación más abiertos y flexibles en el futuro, que garanticen el derecho a la educación inclusiva para todo el alumnado, evitando la segregación y la discriminación. Así como señala el Informe de Seguimiento de la Educación en el Mundo 2020, los países están ampliando su visión de la inclusión en la educación y otorgando a la diversidad un lugar central en sus sistemas, motivados por su compromiso de hacer realidad el derecho a la educación para todas las personas.

\section{Referencias}

Arroyo, M. J. (2013). La Educación Intercultural: un camino hacia la inclusión educativa. Revista de Educación Inclusiva, 6(2),144159.

Azqueta, D.; Gavaldón, G.; Margalef, L. (2007). Educación y desarrollo: ¿capital humano o capital social? Revista de educación, (344), 265-283.

Booth, T.; Ainscow, M. (2002). Guía para la evaluación y mejora de la educación inclusiva. Desarrollando el aprendizaje y la participación en las escuelas. Madrid: Consorcio Universitario para la Educación Inclusiva

Booth, T.; Ainscow, M. (2015). Guía para la educación inclusiva: desarrollando el aprendizaje y la participación en los centros escolares. Madrid: FUHEM / OEI.

Calvo, J. (2008). Sharing Responsibilities and Networking through School Process. Granada: Universidad de Granada.

Carbonell, J. (2015). Pedagogías del siglo XXI: alternativas para la innovación educativa. Barcelona: Octaedro.

Casquero, O. (2013). PLE: una perspectiva tecnológica. En L. Castañeda y J. Adell (Eds.) Entornos personales de aprendizaje: claves para el ecosistema educativo en Red. (7184). Alcoy, España: Marfil. 
CAST (Center for Applied Special Technology). (2018). Diseño Universal para el Aprendizaje. Principios y pautas. Recuperado de https://bit.ly/31K9qOO

CRUE (2017). Formación curricular en diseño para todas las personas. Recuperado de https://bit.ly/2VPPu1y

Díaz, J.; Civís, M. (2011). Redes Socioeducativas promotoras de capital social en la comunidad: un marco teórico de referencia. Cultura y Educación, 23(3), 415-429. DOI: https:// doi.org/10.1174/113564011797330270

Echeita, G.; Ainscow, M. (2011). La educación inclusiva como derecho. Marco de referencia y pautas de acción para el desarrollo de una revolución pendiente. Tejuelo: Revista de Didáctica de la Lengua y la Literatura, (12), 26-45.

Escudero, J. M. (2005). El fracaso escolar. Nuevas formas de exclusión educativa. En J. G. Molina (Coord.), Exclusión social, exclusión educativa. (83-108). Xátiva: Diálogos.

Ferrández, A. (2000). La formación ocupacional en el marco de la formación continua de adultos. En A. Monclús (coord.) Formación y empleo: enseñanza y competencias. (pp. 27-59). Granada: Comares.

Freire, P. (2001). Pedagogía de la indignación. Madrid: Ediciones Morata.

García-Carrasco, J.; Gallardo, B.; García-Manzano, A.; Sánchez, F. (2012). Nuevos modos de aprendizaje en el contexto de la sociedad del conocimiento. En L. García-Aretio (Coord.) Sociedad del conocimiento y educación. (305-346.). UNED: Plasencia.
Global Education Monitoring Report Team. (2020). Global Education Monitoring Report, 2020: Inclusion and Education: All Means All. París: United Nations Educational, Scientific and Cultural Organization. Recuperado de https://bit.ly/36KPxAN

Gutiérrez, E. (2001). La educación en Internet e Internet en la educación como factor supresor de la brecha digital. Congreso la Educación en Internet e Internet en la Educación. Ministerio de Educación, Madrid, 23 de noviembre-12 de diciembre

Lave, J.; Wenger, E. (1991). Situated Learning: Legitimate Peripheral Participation. Cambridge: Cambridge University Press. DOI: https://doi.org/10.1017/CBO9780511815355

Leiva, J. J. (2012). Aportaciones y reflexiones pedagógicas sobre Educación Intercultural: de la diversidad cultural a la cultura de la diversidad. Revista Qurriculum, 25, 55-75.

Levine, T. y Marcus, A. (2010). How the structure and focus of teachers' collaborative activities facilitate and constrain teacher learning. Teaching and Teacher Education, 26(3),389398. DOI: https://doi.org/10.1016/j.tate.2009.03.001

Lois, M. (2011). Estructuración y espacio: la perspectiva de Lugar. Geopolítica (s). Revista de Estudios sobre Espacio y Poder, 1(2), 207-231.

Márquez, A. A. (2018). La Rueda del DUA 2020: actualización de recursos para derribar barreras a la participación. Recuperado de https://bit.ly/3oAGeeh 
Martín, R.; Rinaudo, M. C.; Ordoñez, G. (2012). Cognición situada en contextos de aprendizaje no formales. La experiencia de un curso de guardavidas. En Facultad de PsicologíaUniversidad de Buenos Aires Memorias IV Congreso Internacional de Investigación y Práctica Profesional en Psicología, XIX Jornadas de Investigación, VIII Encuentro de Investigadores en Psicología del Mercosur. (251-254). Buenos Aires: Ediciones de la Facultad de Psicología - Universidad de Buenos Aires. Recuperado de https://bit.ly/3nCca15

Martín-Gutiérrez, Á.; Morales-Lozano, J. A. (2013). La apertura de los centros educativos a su entorno: los centros de educación secundaria. Revista Perspectiva Educacional, 52(1): 68-96. DOI: http://dx.doi.org/10.4151/07189729-Vol.52-Iss.1-Art.119

Mitra, S. (2016). Ambientes de aprendizaje autoorganizados: el enfoque SOLE en las escuelas [Vídeo]. Conferencia presentada en la Escuela Técnica n. ${ }^{\circ} 1$ Ing. Otto Krause, agosto de 2016. Recuperado de https://bit.ly/2HfTN2M

Mitra, S. (julio de 2010). Sugata Mitra y sus nuevos experimentos en autoaprendizaje [Vídeo]. Conferencias TED. Recuperado de https://bit.ly/3IER5CF

Organización de las Naciones Unidas (ONU). (2006). Convención sobre los Derechos de las personas con discapacidad y su Protocolo Facultativo. Ginebra: ONU. Recuperado de https:// bit.ly/2HaRnCv

Pastor, C. A. (2018). Diseño Universal para el Aprendizaje un modelo didáctico para proporcionar oportunidades de aprender a todos los estudiantes. Padres y Maestros/Journal of Parents and Teachers, (374), 21-27. DOI: https://doi.org/10.14422/ pym.i374.y2018.003

Plataforma Estatal por una Escuela Extraordinaria. (2019). Manifiesto por una Escuela Extraordinaria. Recuperado de https://bit. ly $/ 2 \mathrm{HdlsBm}$
Santos, M. A. (Ed.) (2009). Políticas educativas y compromiso social: el progreso de la equidad y la calidad. Madrid: Octaedro.

Slee, R. (2012): La escuela extraordinaria. Exclusión, escolarización y educación inclusiva. Madrid: Morata.

Tonucci, F. (2017). A modo de introducción... La diversidad como valor en una escuela que cambia. Aula Abierta, 46(2), 9-12. DOI: https://doi.org/10.17811/rifie.46.2017.9-12

Unesco. (2008). La educación inclusiva: el camino hacia el futuro. Conferencia Internacional de Educación. Centro Internacional de Conferencias, Ginebra, 25-28 de noviembre. Recuperado de https://bit.ly/2HdlRUo

Unesco. (2016). Educación 2030: Declaración de Incheon y Marco de Acción para la realización del Objetivo de Desarrollo Sostenible 4: Garantizar una educación inclusiva y equitativa de calidad y promover oportunidades de aprendizaje permanente para todos. Recuperado de https://bit.ly/3IFSzwE

Unesco. (2020a). Impacto del COVID-19 en la educación. Recuperado de https://bit.ly/2ILxqCl

Unesco. (2020b). Coalición Mundial para la Educación COVID-19. Recuperado de https://bit.ly/38SNMnN

Vega, A. (2010). La educación inclusiva: un deber de justicia. Intersticios: Revista Sociológica de Pensamiento crítico, 4(1), 57-77.

Villa, N. (2015). La universidad ante la riqueza de la diversidad funcional: retos y desafíos para una formación on line de calidad y accesible. En L. Bengocheas; C. Varela; A. Miñán (eds.), Formación virtual inclusiva y de calidad para el siglo XXI. Actas del VI Congreso Internacional sobre Calidad y Accesibilidad de la Formación Virtual (133-139). Granada: Universidad de Granada. Recuperado de https://bit.ly/32TgdOI 